\title{
Dynamic Load Management and Optimum Sizing of Stand- Alone Hybrid PV/Wind System
}

\author{
E. Kaplani ${ }^{*}$, P. Ntafogiannis, K. Pappas, N. Diamantopoulos \\ R.E.S. Laboratory, Mechanical Engineering Dept., Technological Educational Institute of Western Greece \\ M. Alexandrou 1, Koukouli 26 334, Patra, Greece \\ *Correspondence: ekaplani@teipat.gr
}

\begin{abstract}
Simulation algorithms for the sizing of stand-alone hybrid PV/Wind systems are a powerful tool in evaluating the optimum configuration that would cover the energy demand with a predefined reliability level at the lowest cost. Several parameters such as the interval of the simulation (day, day-night, hourly) and the consumption profile may significantly affect the optimum configuration. This paper examines the effect of these parameters within an optimum sizing simulation algorithm developed. The effect of these parameters was particularly evident at low battery capacities, which involve optimum configurations resulting in minimum cost. Furthermore, shift-able loads in the hourly-based weekly profile assumed in this study were identified, and a dynamic load management functionality was developed. In this approach, loads that could be shifted through time were dynamically allocated during periods of excess energy production by the hybrid PV/Wind system. The results showed an increase in system reliability from $95 \%$ to $97 \%$ when load shifting was introduced. Finally, sizing the system for only the static (non-shift-able loads) proved to withstand the addition of the extra shift-able loads while retaining the $95 \%$ reliability level when the load management functionality was introduced. Thus, a smaller installation with lower cost is achieved.
\end{abstract}

Keywords: Hybrid PV/Wind system, Optimum sizing, Load shifting, Intelligent Energy Buildings PACS: $88.40 .-\mathrm{j}, 88.50 .-\mathrm{k}, 88.05 .-\mathrm{b}$

\section{INTRODUCTION}

Stand-alone hybrid PV/Wind systems present a promising solution to cost-effectively cover the energy needs in remote locations, and are gaining increasing attention the recent years in building applications. In off-grid building applications, the optimum sizing of hybrid PV/Wind systems is of particular importance in order to provide with a cost-effective and reliable solution to meet the occupants energy demand throughout the year. Optimum sizing algorithms for stand-alone systems have been proposed based on the loss of load probability (LLP) and loss of power supply probability (LPSP) [1-3], stochastic modeling [4-6], Neural Networks [7], and others, with the minimum cost [8]. The economic analysis often involves the estimation of the levelised cost of energy (LCE) [1,3,9] taking into account the initial capital cost of the hybrid system, maintenance and replacement costs in the lifetime of the system. A review of optimum sizing approaches for hybrid PV/wind systems is provided in [10]. The optimization algorithms may be based on historical solar radiation and wind data, or modeled, stochastic data. Some size optimization algorithms simulate system performance on an hourly basis, while other on a day-night, or daily basis. The current study evaluates the impact in the optimum sizing configuration of a hybrid PV/wind system brought by the different simulation intervals, and examines also the impact of the energy consumption behavior, i.e. using different hourly load profiles based on day, night and mixed weekly consumption. The concept of dynamic load management is then applied on the resulting optimum configuration of the hybrid PV/Wind system, as an application to intelligent energy buildings, whereby loads that may be shifted through time are dynamically allocated during periods of excess energy production by the PV generator and wind turbine. The impact of dynamic load management for a more reliable and cost-effective system operation is assessed.

\section{OPTIMUM SIZING OF HYBRID PV/WIND SYSTEM}

The hybrid PV/wind system considered in the simulation algorithm developed comprises of a PV generator with crystalline silicon PV modules oriented towards South and inclined at a $30^{\circ}$ angle (optimum angle for the city of Athens, Greece) a wind turbine of $0.5 \mathrm{~kW}$ and $1 \mathrm{~kW}$, and a battery system with $80 \%$ depth-of-discharge. The one year 
hourly solar radiation and wind speed data for the city of Athens used in this study are displayed in Fig. 1. The hourly solar irradiance is converted to the $30^{\circ}$ angle of inclination and the wind speed data translated to $15 \mathrm{~m}$ at hubheight using the power law, eq.(1), [11].

$$
\frac{v}{v_{\text {ref }}}=\left(\frac{z}{z_{\text {ref }}}\right)^{n}
$$

where $v$ is the wind speed at hub-height $\mathrm{z}, v_{\text {ref }}$ the wind speed measured at the reference height $\mathrm{z}_{\text {ref }}$ and the exponent $\mathrm{n}$ considered equal to $1 / 7$.

In modeling the PV system, the PV generator power output is estimated based on eq. (2). $\mathrm{I}_{\mathrm{T}}$ is the solar irradiance on the inclined PV plane, $\mathrm{N}$ the number of PV modules and $\mathrm{A}_{\mathrm{m}}$ the area of each module. The PV efficiency $\eta_{\mathrm{PV}}$ is converted to the operating conditions at each hour based on eq. (3).

$$
\begin{aligned}
& P_{P V}=\eta_{P V} \cdot N \cdot A_{m} \cdot I_{T} \\
& \eta_{P V}=\eta_{P V, S T C} \cdot\left(1+\gamma \cdot\left(T_{P V}-T_{P V, \text { ref }}\right)+\delta \cdot \ln \left(\frac{I_{T}}{I_{T, \text { ref }}}\right)\right.
\end{aligned}
$$

where $\eta_{\mathrm{PV}, \mathrm{STC}}$ is the PV efficiency at Standard Test Conditions $\left(\mathrm{I}_{\mathrm{T}, \mathrm{ref}}=1000 \mathrm{~W} / \mathrm{m}^{2}, \mathrm{~T}_{\mathrm{PV}, \mathrm{ref}}=25^{\circ} \mathrm{C}\right.$, Air Mass 1.5$), \gamma$ is the temperature coefficient for power considered $-0.5 \% /{ }^{\circ} \mathrm{C}$ and $\delta$ the solar irradiance coefficient equal to 0.085 for single crystalline and 0.11 for poly-crystalline modules [12].

The PV temperature was estimated based on eq. (4).

$$
T_{P V}=T_{a}+f \cdot I_{T}
$$

Coefficient $f$ which depends on the type of PV installation (free standing, BIPV, etc.), the PV inclination, the wind velocity and direction, the pattern of air flow along the PV panel, etc, was considered equal to $0.03 \mathrm{~m}^{2}{ }^{\circ} \mathrm{C} / \mathrm{W}$. Precise modelling of $f$ based on the hourly values of wind velocity and wind direction, and the module orientation and inclination may be included as provided in [13].

The wind turbine power output was modelled based on the manufacturer power curve data of the wind turbine. A $0.5 \mathrm{~kW}$ and a $1 \mathrm{~kW}$ wind turbine were considered, see power curves provided in Fig.2. The power output of the wind turbine may be approximated through cubic spline interpolation, as proposed in [14]. Similarly to this and as in [15], cubic polynomials were fitted on segments of the power curve to provide best fit. Here, eq.(5) is applied with $n$ equal to 5 .

$$
P_{w}=\left\{\begin{array}{cc}
0 & v<v_{c i} \\
a_{1} v^{3}+b_{1} v^{2}+c_{1} v+d_{1} & v_{c i} \leq v \leq v_{1} \\
a_{2} v^{3}+b_{2} v^{2}+c_{2} v+d_{2} & v_{1}<v \leq v_{2} \\
a_{n} v^{3}+b_{n} v^{2}+c_{n} v+d_{n} & \begin{array}{c}
\cdots \\
v_{n-1}<v \leq v_{c o} \\
v>v_{c o}
\end{array}
\end{array}\right.
$$

$\mathrm{P}_{\mathrm{w}}$ is the power output of the wind turbine at wind speed $v$ at hub-height, $v_{c i}$ and $v_{c o}$ the cut-in and cut-out wind speeds.

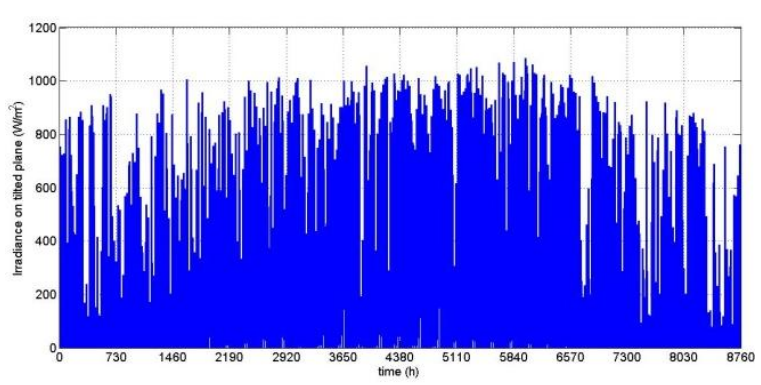

(a)

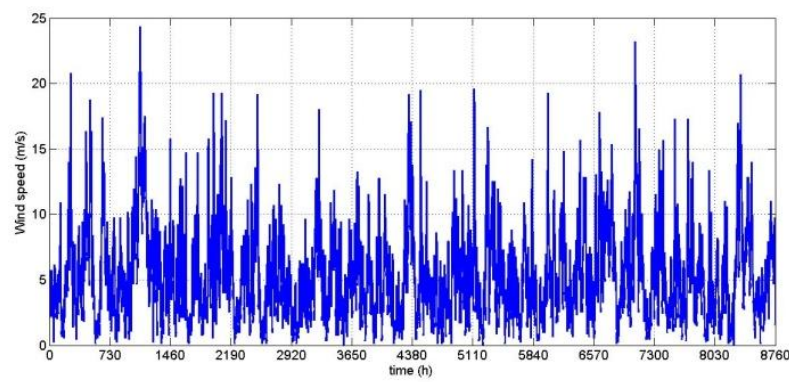

(b)

FIGURE 1. (a) Solar irradiance on PV plane inclined at $30^{\circ}$ angle, (b) wind velocity at hub-height $15 \mathrm{~m}$, at the city of Athens. 


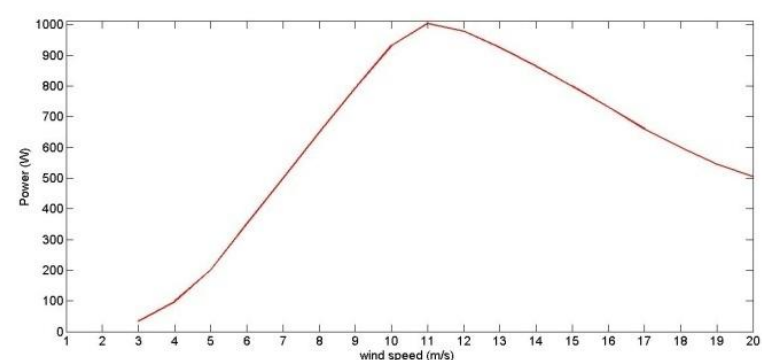

(a)

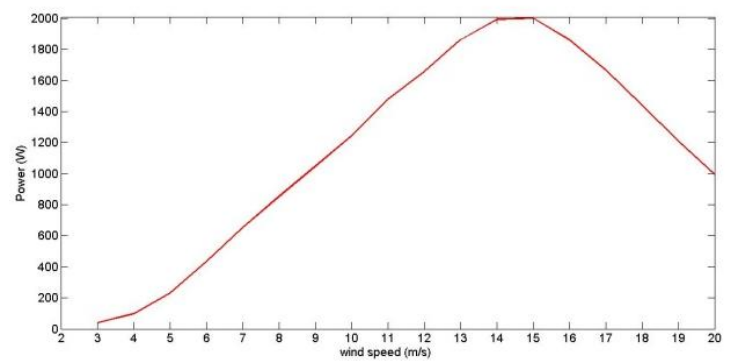

(b)

FIGURE 2. Power curve of (a) $0.5 \mathrm{~kW}$ wind turbine, (b) $1 \mathrm{~kW}$ wind turbine.

In the hourly simulation the remaining energy produced by the PV and the wind turbine at hour $\mathrm{t}$ that is not consumed by the loads is stored in the battery system as long as the state-of-charge (SOC) of the battery has not reached its higher limit. At hours where the energy demand is greater than the energy produced, the battery system provides the remaining energy to cover the energy needs through a discharging process as long as the SOC of the battery has not dropped below the 1-DOD (depth-of-discharge) with DOD considered $80 \%$. The charging and discharging state of the battery is given by eq.(6) and eq.(7) respectively [2]. $\sigma$ is the hourly self-discharge rate.

$$
\begin{aligned}
& E_{b a t}(t)=E_{b a t}(t-1) \cdot(1-\sigma)+\left(E_{p v}(t)+E_{w}(t)-E_{L}(t) / \eta_{i n v}\right) \cdot \eta_{b a t} \\
& E_{b a t}(t)=E_{b a t}(t-1) \cdot(1-\sigma)-\left(E_{L}(t) / \eta_{i n v}-E_{p v}(t)-E_{w}(t)\right)
\end{aligned}
$$

The optimum sizing is evaluated through the LPSP [2], with the required LPSP set at 5\% for operation with noncritical loads, eqs.(8)-(9), and the minimum cost evaluated through the LCE [9].

$$
\begin{aligned}
& \operatorname{LPSP}=\frac{\sum_{t=1}^{8760} \operatorname{LPS}(t)}{\sum_{t=1}^{8760} E_{L}(t)} \\
& \operatorname{LPS}(t)=\left(E_{L}(t)-\left(E_{P V}(t)+E_{w}(t)+C_{\text {bat }}(t-1)-(1-D O D) \cdot C_{\text {bat }, \text { nom }}\right) \cdot \eta_{\text {inv }}\right)
\end{aligned}
$$

An hourly based weekly load profile was used for the simulation, provided in Fig. 3 presented as the hourly percentage of the total daily consumption. The total daily consumption had seasonal variations and was considered $10 \mathrm{kWh} /$ day in Winter, $8.5 \mathrm{kWh} /$ day in Spring and 13kWh/day in Summer.

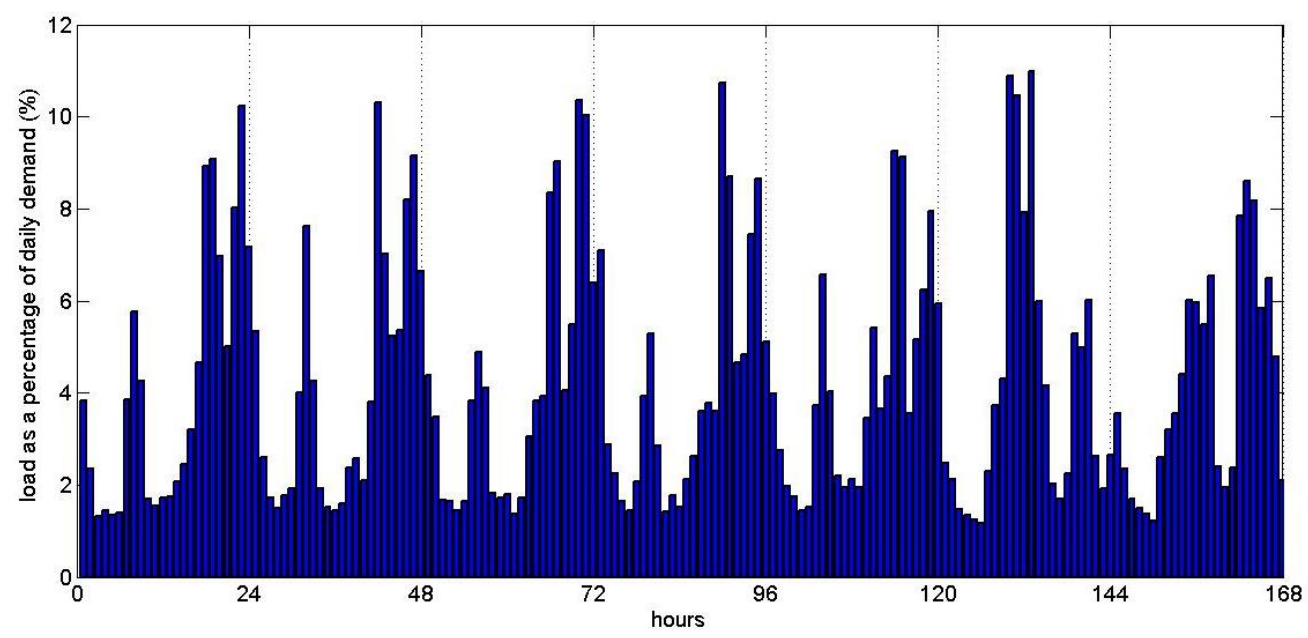

FIGURE 3. Hourly-based weekly load profile. The hourly value is presented as a percentage of the total daily consumption $(10 \mathrm{kWh} /$ day in Winter, $8.5 \mathrm{kWh} /$ day in Spring, $13 \mathrm{kWh} /$ day in Summer) 


\section{Optimum configuration of hybrid PV/wind system}

The optimum sizing results are presented in Fig. 4. The optimum configuration with LPSP equal to 5\% is given by the $0.5 \mathrm{~kW}$ wind turbine, PV power of $2100 \mathrm{Wp}$ and a total battery capacity of $917 \mathrm{Ah}$ resulting in the lowest cost of $0.206 € / \mathrm{kWh}$. Smaller PV system with higher battery capacity could provide the same reliability but at a higher cost, mainly due to the higher replacement cost of the batteries, replaced twice during the 25 year expected lifetime of the system. Due to the relatively low wind speed profile, the optimum configuration with the lowest cost is provided using the $0.5 \mathrm{~kW}$ wind turbine, consequently resulting in lower excess energy (33\%), see Fig.5.

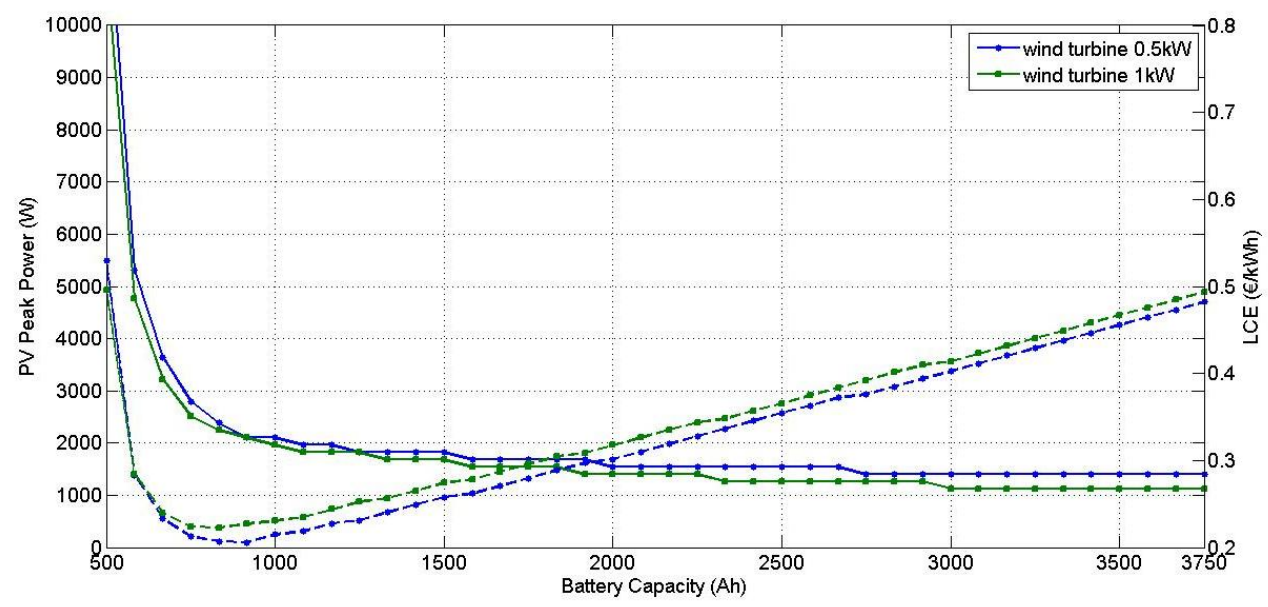

FIGURE 4. PV peak power vs battery capacity for wind turbine $0.5 \mathrm{~kW}$ and $1 \mathrm{~kW}$ obtained for LPSP $5 \%$ (solid lines), and corresponding LCE values for these configurations (dashed lines).

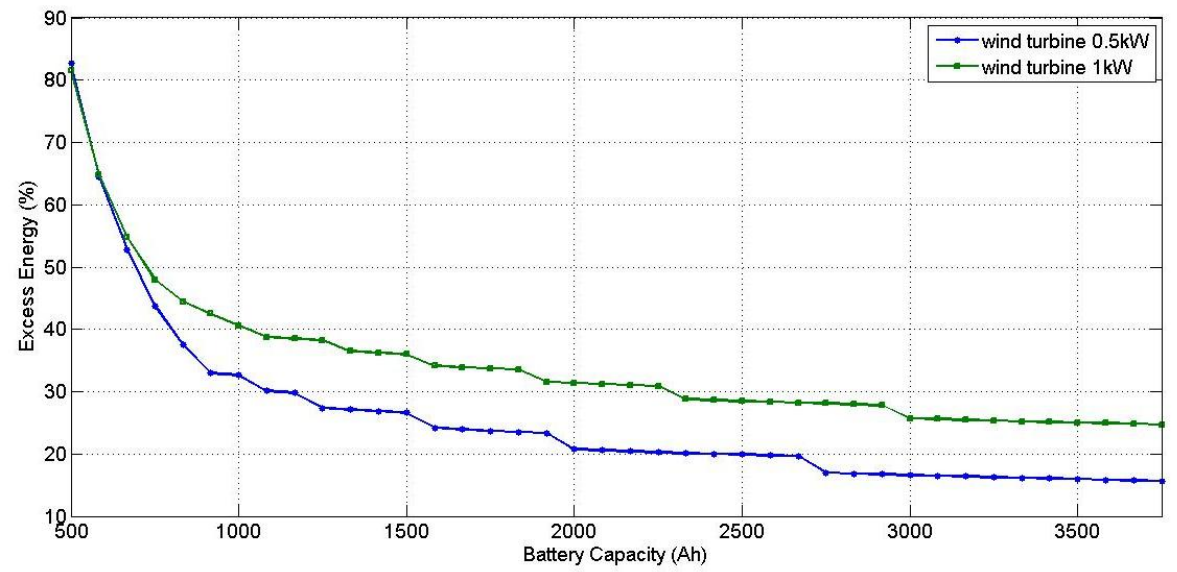

FIGURE 5. Excess energy in percentage of the total yearly energy produced by the hybrid system with the $0.5 \mathrm{~kW}$ and the $1 \mathrm{~kW}$ wind turbine.

\section{Effect of simulation interval and load profile on the optimum configuration}

The effect of the simulation interval on the optimum configuration was examined through 3 different approaches. The main simulation approach estimates the energy produced by the hybrid system, stored in the battery system and consumed by the loads at hourly intervals (hourly simulation), a second simulation approach at daily intervals (day simulation) and a third approach at the end of the day and at the end of the night (day-night simulation). The daynight simulation and the day-based simulation underestimate the sizing of the system, which is provided more accurately by the hourly approach. This underestimation is significant at low battery capacities, see Fig. 6 . 
Besides the hourly-based weekly load profile used where the consumption profile changes within the week, the simulation was also executed for two hourly-based daily profiles that were kept constant within the week. The one was based on higher load demand during the night period and the other on higher load demand during the daytime. The night-based profile used was the hourly profile that corresponds to the first day of the weekly profile shown in Fig. 3 (hours 0-24), and the day-based profile used was the hourly profile that corresponds to the 6th day of the weekly profile in Fig.3 (hours 120-144). All profiles were adapted to the seasonal demand earlier stated. The results show that when a load profile based on day-time consumption exists, a smaller system is required particularly for lower battery capacity configurations, as displayed in Fig.7. On the contrary, when the profile is based on night-time consumption, a slightly larger system is required than that for a mixed weekly consumption profile (of Fig. 3), particularly for lower battery capacity configurations, corresponding to lower system cost, see Fig.7.

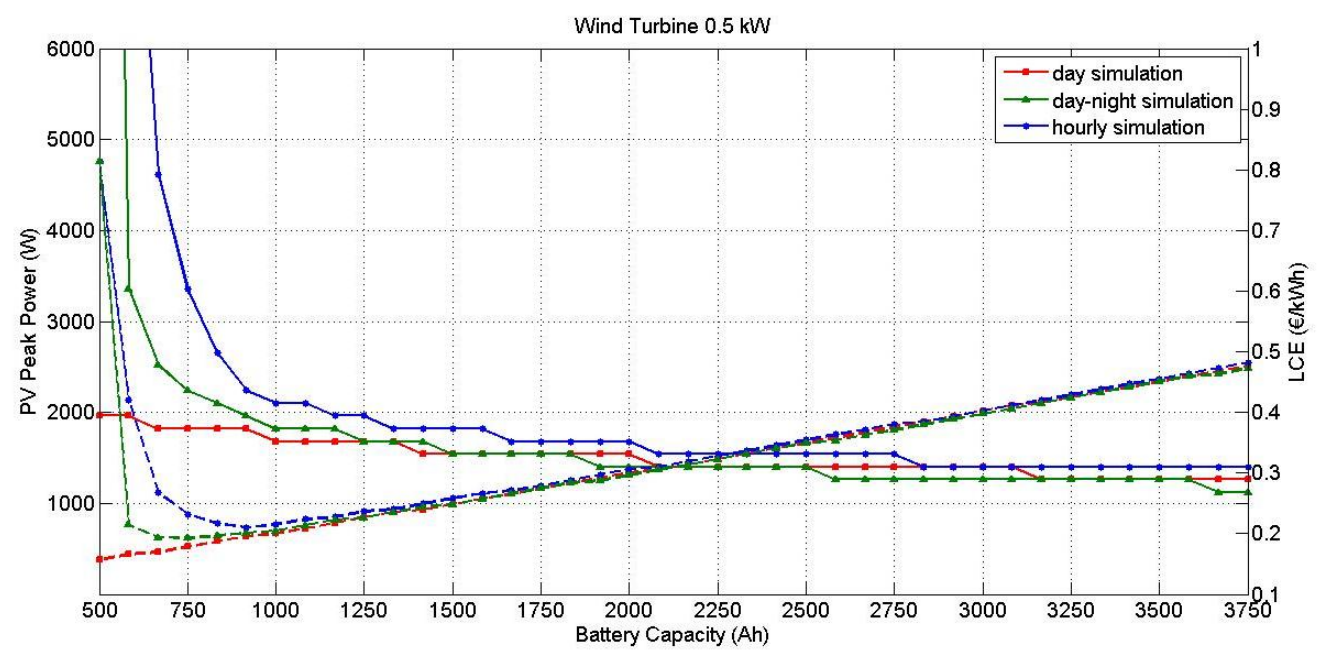

FIGURE 6. PV peak power vs battery capacity for wind turbine $0.5 \mathrm{~kW}$ obtained for LPSP $5 \%$ and the different simulation approaches: hourly, day-night, day (solid lines), and corresponding LCE values for these configurations (dashed lines).

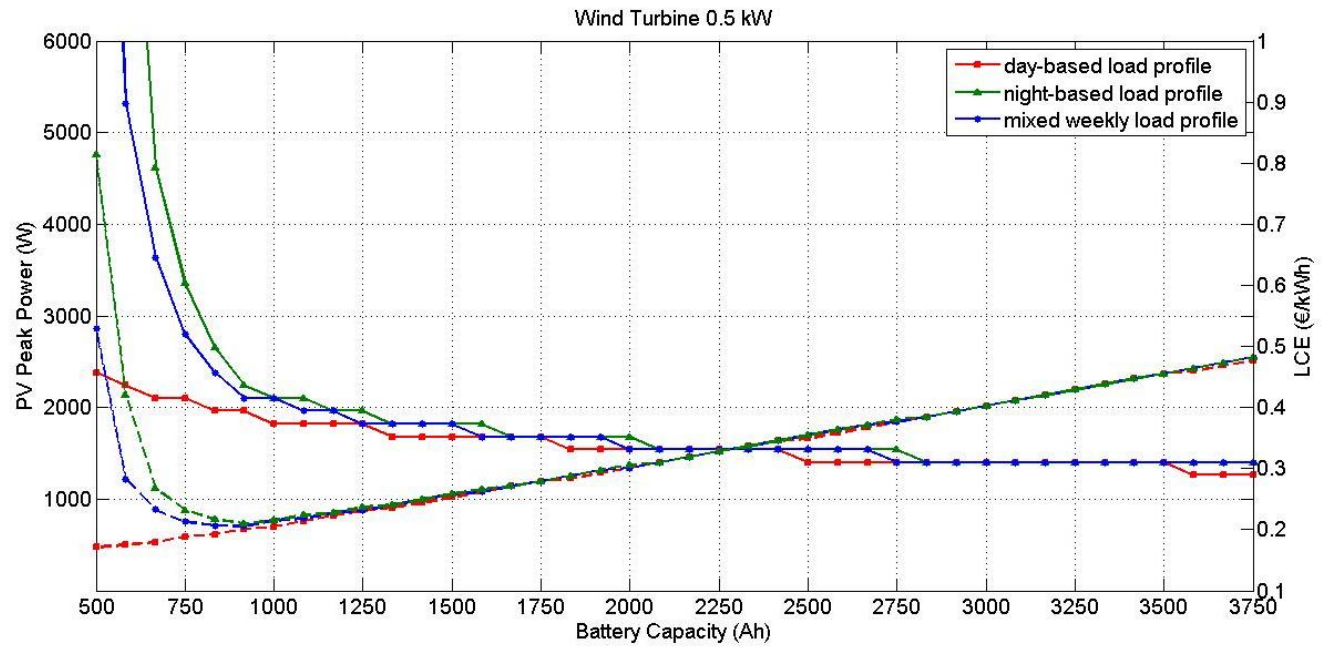

FIGURE 7. PV peak power vs battery capacity for wind turbine $0.5 \mathrm{~kW}$ obtained for LPSP $5 \%$ and the different consumption profiles: day-based consumption, night-based consumption, mixed weekly consumption profile (solid lines), and corresponding LCE values for these configurations (dashed lines). 


\section{DYNAMIC LOAD MANAGEMENT}

A dynamic load management functionality was further developed in order to take advantage of the amount of excess energy produced by the system and to minimize further system cost. Loads that could be shifted in time were identified, such as washing machine (sh. load 1), dish washer (sh. load 2), clothes iron (sh. load 3), vacuum cleaner (sh. load 4), and appear in red in the weekly load profile provided in Fig. 8. According to user preferences, the priority in operating the shift-able loads, the number of times a load is required to operate within the week, and the maximum period allowed for delaying load operation are preset. Here, loads 1-3 were set to operate twice a week and load 4 once a week, while their shifting threshold was relaxed to a period of one week with the possibility of continuous operation in load 1 and 3 . Whenever excess energy is produced by the hybrid system, after providing for the static (non-shift-able) loads and fully charging the battery, the remaining excess energy if sufficient is used to power any shift-able loads. In case the excess energy produced at a particular hour is not enough to cover the demand of a shift-able load, and its maximum predefined shifting period has been reached then the load is served at the next day-time instance with the battery contributing to the supply of the required energy.

The system performance for the optimum sizing configuration selected to cover the total energy demand, is shown in Fig. 9, where the energy produced by the wind turbine and the PV modules, the SOC of the battery system, the total load demand and the excess energy produced after powering the shift-able loads is presented for a period during Spring. By introducing load shifting functionality the system resulted in a higher reliability level of $97 \%$ compared to the previous $95 \%$.

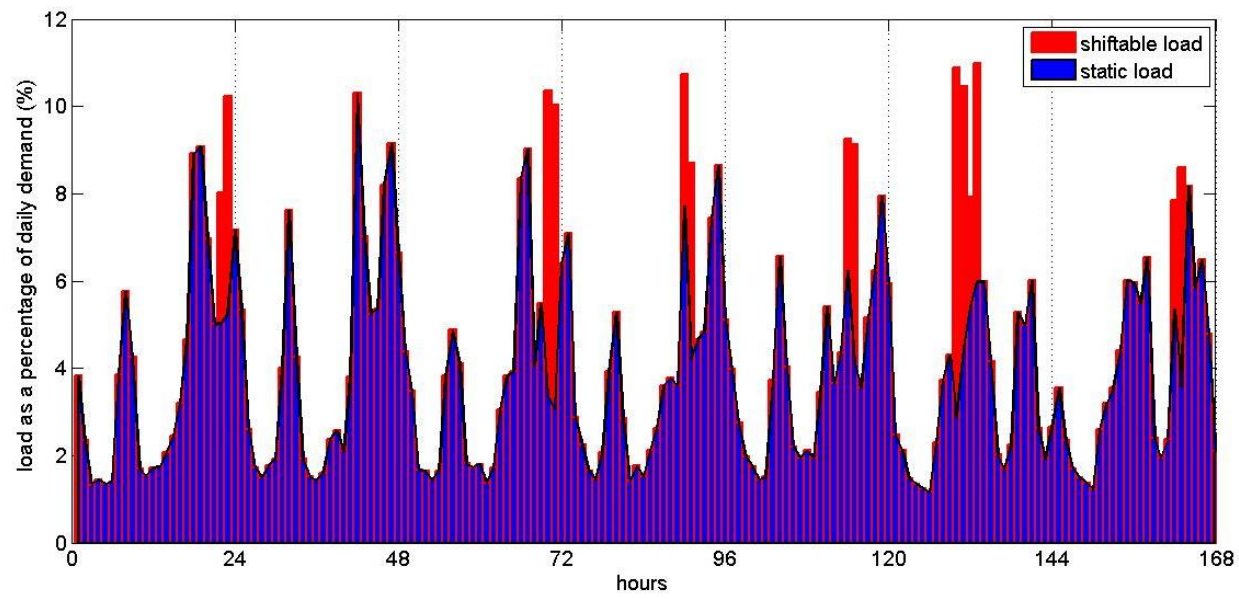

FIGURE 8. Shift-able loads and static (non-shift-able) loads in the weekly profile.
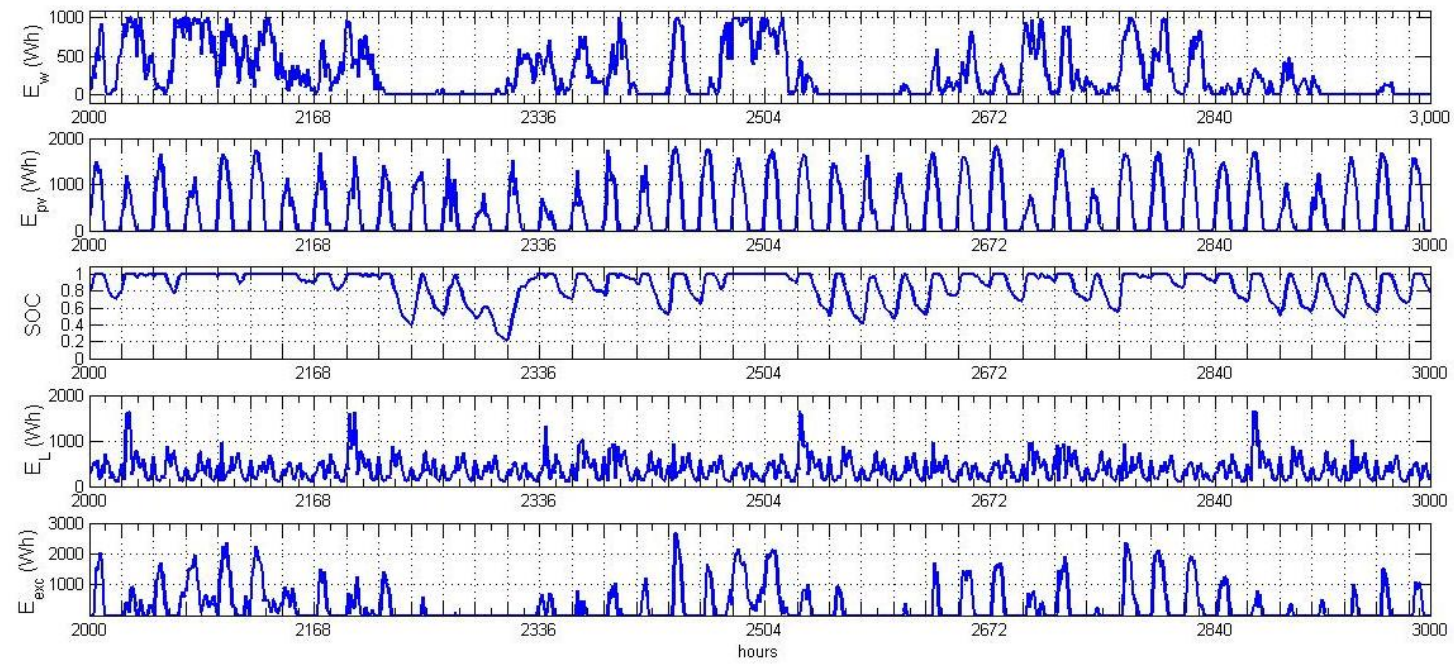

FIGURE 9. Energy produced by the wind turbine $\left(\mathrm{E}_{\mathrm{w}}\right)$ and the PV modules $\left(\mathrm{E}_{\mathrm{PV}}\right)$, SOC of battery system, total energy consumed by the loads $\left(\mathrm{E}_{\mathrm{L}}\right)$ and excess energy produced $\left(\mathrm{E}_{\mathrm{exc}}\right)$ after providing for the shift-able loads. 
To provide a more cost-effective solution, the hybrid PV/wind system was sized for the static (non-shift-able) loads alone with the optimum simulation algorithm described above, thus for a winter's $63.35 \mathrm{kWh}$ load per week instead of the initial $70 \mathrm{kWh}$. The optimum configuration resulted in the $0.5 \mathrm{~kW}$ wind turbine, a PV power $2100 \mathrm{~W}$ and a smaller battery capacity of 750Ah. Fig. 10 shows the SOC of the battery system and the excess energy before and after operating the shift-able loads, along with the ON/OFF power state of the loads, the total energy consumed and the subsequent excess energy. The shift-able loads are dynamically started when sufficient excess energy is produced and the state of charge of the battery is full.

In this scenario the hybrid system is sized to cover only the static (non-shift-able) loads, while the introduction of the shift-able loads (approximately $10 \%$ of the total weekly loads) sustains system reliability level at $95.8 \%$. Thus, a lower cost for the installation is achieved.

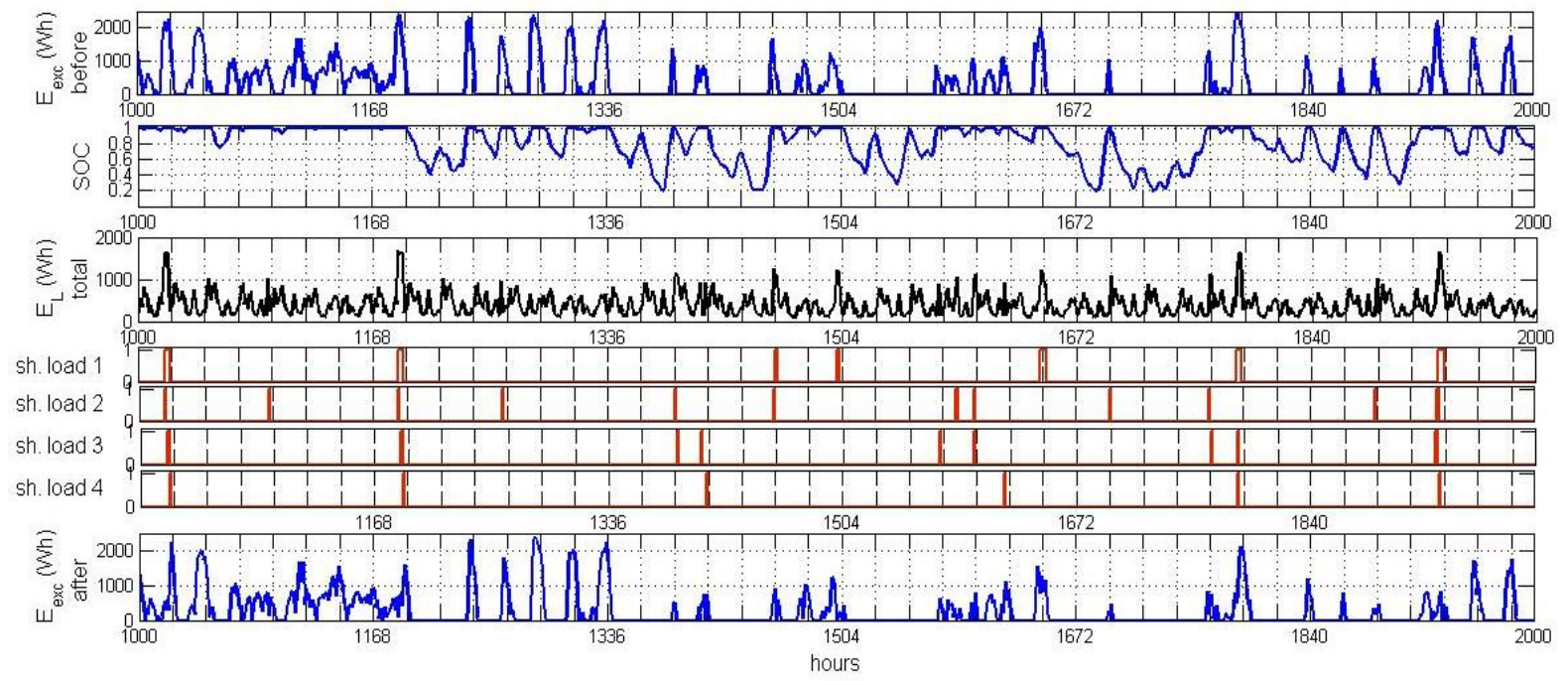

FIGURE 10. Excess energy $\left(\mathrm{E}_{\text {exc }}\right)$ and SOC of the battery system before the shift-able loads are started, total energy consumed $\left(\mathrm{E}_{\mathrm{L}}\right)$ including the shift-able loads, the ON/OFF power state of the shift-able loads, the remaining excess energy after the shiftable loads are supplied with energy.

\section{CONCLUSIONS}

A simulation algorithm for the optimum sizing of stand-alone hybrid PV/Wind system was developed. The simulation was executed for hourly solar and wind data for the city of Athens, using an hourly-based weekly profile. The optimum sizing configuration for the minimum cost resulted in small battery capacity. The effect of the simulation interval, such as hourly, day, day-night, was also tested and was found to significantly affect the optimum configuration at low battery capacities. Furthermore, the effect of the hourly consumption profile whether based on night consumption, day consumption or mixed consumption during the week, also showed to affect the optimum configuration at low battery capacities, with the profile based on daytime consumption to lead to smaller system configuration requirements.

Finally a load management functionality was introduced in the optimum configuration selected. Loads that could be shifted through time were dynamically allocated during periods of excess energy production by the hybrid PV/Wind system. The results showed an increase in system reliability from $95 \%$ to $97 \%$ when load shifting was introduced. In addition, sizing the system for only the static (non-shift-able loads) proved to withstand the addition of the extra shift-able loads sustaining the same level of reliability $95 \%$ when the load management functionality was introduced. Thus a smaller installation and lower cost is achieved.

Further work is currently being carried out in the predictive management of loads incorporating hourly solar radiation and wind speed prediction.

\section{REFERENCES}

1. H. Yang, L. Lu, W. Zhou, "A novel optimization sizing model for hybrid solar-wind power generation system", Solar Energy 81, 76-84 (2007).

2. B. Ai, H. Yang, H. Shen, X. Liao, "Computer-aided design of PV/wind hybrid system", Renewable Energy 28, $1491-1512$ (2003). 
3. A. Rajendra Prasad, E. Natarajan, "Optimization of integrated photovoltaic-wind power generation systems with battery storage", Energy 31, 1943-1954 (2006).

4. T. Markvart, A. Fragaki, J.N. Ross, "PV system sizing using observed time series of solar radiation", Solar Energy 80, 46-50 (2006).

5. A. Balouktsis, et al., "Sizing stand-alone photovoltaic systems. International Journal of Photoenergy, 1-8 (2006), doi:10.1155/ijp/2006/73650.

6. E. Kaplani, S. Kaplanis, "A stochastic simulation model for reliable PV system sizing providing for solar radiation fluctuations", Applied Energy 97, 970-981 (2012).

7. A. Mellit, S. Kalogirou, "Artificial intelligence techniques for photovoltaic applications: A review", Progress in Energy and Combustion Science 34, 574-632 (2008).

8. J.K. Kaldellis, D. Zafirakis, K. Kavadias, "Minimum cost solution of wind-photovoltaic based stand-alone power systems for remote consumers", Energy Policy 42, 105-117 (2012).

9. S. Diaf, G. Notton, M. Belhamel, M. Haddadi, A. Louche, "Design and techno-economical optimization for hybrid PV/wind system under various meteorological conditions", Applied Energy 85, 968-987 (2008).

10. W. Zhou, C. Lou, Z. Li, L. Lu, H. Yang, "Current status of research on optimum sizing for stand-alone hybrid solar-wind power generation systems", Applied Energy 87, 380-389 (2010).

11. C.G. Justus, A. Mikhail (1976). Height variation of wind speed and wind distributions statistics. Geophysical Research Letters 3(5), 261-264.

12. A.J. Anderson, "Photovoltaic translation equations: A new approach", in Final Subcontract Report. NREL/TP-411-20279, 1996.

13. E. Kaplani, S. Kaplanis, "Thermal modelling and experimental assessment of the dependence of PV module temperature on wind velocity and direction, module orientation and inclination", Solar Energy 107, 443-460 (2014).

14. S. Diaf, D. Diaf, M. Belhamel, M. Haddadi, A. Louche, "A methodology for optimal sizing of autonomous hybrid PV/wind system", Energy Policy 35, 5708-2718 (2007).

15. F.O. Hocaoglu, O.N. Gerek, M. Kurban, "A novel hybrid (wind-photovoltaic) system sizing procedure", Solar Energy 82, 2019-2028 (2009). 